\title{
Carnitine-Acylcarnitine Translocase Deficiency
}

National Cancer Institute

\section{Source}

National Cancer Institute. Carnitine-Acylcarnitine Translocase Deficiency. NCI Thesaurus.

Code C133086.

An autosomal recessive condition caused by mutation(s) in the SLC25A20 gene, encoding mitochondrial carnitine/acylcarnitine carrier protein. It is characterized by cardiomyopathy, skeletal muscle damage, and liver dysfunction that results from derangement of long-chain fatty acid oxidation. 